\title{
Hormonal and genetic regulation of axillary bud outgrowth in Chrysanthemum morifolium during floral initiation
}

Robrecht Dierck ${ }^{1}$, Ellen De Keyser ${ }^{1}$, Jan De Riek ${ }^{1}$, Emmy Dhooghe ${ }^{1}$, Johan Van Huylenbroeck $^{1}$, Els Prinsen ${ }^{2}$, Dominique Van Der Straeten ${ }^{3}$

${ }^{1}$ Institute for Agricultural and Fisheries Research (ILVO)

Caritasstraat 21, Melle, Belgium

${ }^{2}$ University of Antwerp, Groenenborgerlaan 171, Antwerp, Belgium

${ }^{3}$ Laboratory of Functional Plant Biology, Department of Physiology, Ghent University, K.L. Ledeganckstraat 35, Ghent, Belgium

Keywords: Chrysanthemum morifolium, axillary bud outgrowth, auxin, cytokinin, hormone measurement, gene expression

\begin{abstract}
Shoot branching or the outgrowth of axillary buds is a crucial aspect in the production and breeding of Chrysanthemum sp. The outgrowth of axillary buds is inhibited by the shoot apex through apical dominance. Central to this regulation is the interaction of the plant hormones auxin, cytokinin and strigolactone. In this research we aim to study the hormonal and genetic regulation of apical dominance and axillary bud outgrowth in a cut flower cultivar of Chrysanthemum. For this we combined measurements of axillary bud outgrowth with UPLC-MS/MS quantification of auxin and cytokinin levels and RT-qPCR analysis of two genes involved in the regulation of branching. The cultivar used displayed apical dominance during vegetative growth. A release from apical dominance occurs during floral initiation with outgrowth and elongation of shoots under the apex. The outgrowth of axillary buds after release from apical dominance was preceded by decreased auxin levels in the shoot apex, axillary buds and stem and increased cytokinin levels in the axillary buds. RT-qPCR showed a decreased expression of the local bud outgrowth regulator $B R C 1$ preceding the release from apical dominance at the time of floral initiation.
\end{abstract}

\section{INTRODUCTION}

Chrysanthemum morifolium Ramat., also known as the florist's chrysanthemum and the garden mum, is an economically important horticultural crop. A variety of shapes is available on the market, both as cut flower and potted plant. Shoot branching is a crucial factor in breeding and production of chrysanthemum for obtaining a desirable architecture. Shoot branching has a complex regulation. Externally, shoot branching is influenced by light, temperature nutrients and pruning; internally it is regulated by the interaction of plant hormones (Domagalska and Leyser 2011).

Important in the control of axillary bud outgrowth is apical dominance, the control exerted by the vegetative shoot apex over the outgrowth of axillary buds (Cline, 1991). Removal of the shoot apex or transition to the generative growth stage releases apical dominance and allows lateral bud outgrowth. Central to the regulation of axillary bud outgrowth is the interaction of three classes of plant hormones: auxins, cytokinins and 
strigolactones. Auxins are the plant hormones, since long associated with apical dominance (Thimann et al., 1934). Auxins are produced by young expanding leaves at the shoot apex and transported through the stem towards the roots. This polar auxin transport does not deliver tothe axillary buds, which indicates an indirect effect of auxin on axillary buds (Leyser, 2009). Auxin has been shown to up regulate strigolactone biosynthesis (Hayward et al., 2009). The strigolactones inhibit bud outgrowth directly (Waldie et al., 2014) and also act together with auxins in inhibiting the axillary buds (Shinohara et al., 2013). Auxins also downregulate cytokinin biosynthesis and upregulate cytokinin degradation. This decreases the cytokinin levels that are needed to promote bud outgrowth (Müller and Leyser, 2011) and to counteract the action of auxins and strigolactones in inhibiting axillary buds (Dun et al., 2012).

The complex hormonal interactions of shoot branching regulation are directed by the activity of several genes involved in the homeostasis, transport and perceptions of the plant hormones. In chrysanthemum only a few regulatory genes have been described. IPT (isopentenyltransferase) is a key gene in cytokinin biosynthesis. Overexpression of the IPT gene in chrysanthemum induces an enhanced branching phenotype (Khodakovskaya et al., 2009). Branchedl (BRC1) is a local transcription factor in axillary buds. In chrysanthemum, high $B R C 1$ expression was shown in inhibited axillary buds and the gene was downregulated in activated buds (Chen et al., 2013). Strigolactone regulating genes $M A X 4$ and MAX2 and the lateral suppressor like $(\mathrm{Lsl})$ gene have also been described in the analysis of shoot branching in chrysanthemum (Liang et al., 2010; Dong et al., 2013; Jiang et al., 2009).

In this work we aim to study the regulation of axillary bud outgrowth in a chrysanthemum cut flower cultivar during the transition from vegetative to generative growth accompanied by a release from apical dominance. The change of endogenous hormones in apex and axillary buds during vegetative growth and floral transition has already been studied in chrysanthemum cut flower cultivars by Jiang et al. (2012). In this study, measurements of axillary bud outgrowth is compared with hormone measurements in apex, stem and axillary bud samples. Furthermore the expression of two genes involved in the regulation of branching, $B R C 1$ and $I P T 3$, is used to integrate the effects at the molecular level.

\section{MATERIAL AND METHODS}

\section{Plant Material and growth conditions}

The Chrysanthemum morifolium cultivar used in this study is a split-type chrysanthemum. This means that the plant initiates floral transition at the shoot apex after forming a certain number of leaves during vegetative growth. 100 cuttings were rooted in trays for three weeks in May and June 2014. After rooting, the plants were put in 3L pots in standard potting soil and placed with $20 \mathrm{~cm}$ spacing under LD conditions in the greenhouse at $20^{\circ} \mathrm{C}$ in June. Two weeks after repotting measurements and sampling started in the first week of July. Axillary bud/shoot length and total plant height was measured weekly on 10 plants during 5 subsequent weeks, further referred to as week1, week2, week3, week4 and week5.

\section{Sampling Plant material}

The remaining 90 plants were sampled in week 1 and in week2. At both dates 3 replicated samples were taken, and each replicate consisted of 15 plants. In week1 the 
shoot apices, i.e. the top parts of the stem above the first fully unfolded leaf, were harvested. The 30 nodal positions under the apex were also sampled. For each nodal position the axillary buds and stems were sampled separately. In week 2 the apices and 15 nodal positions under the apex were sampled in the same way. The lateral buds below this zone had already grown out and were not sampled at week2. Samples were immediately frozen in liquid nitrogen and stored at $-80^{\circ} \mathrm{C}$. Samples were pooled based on the information of axillary bud outgrowth as shown in Figure 1. Nodal positions 37 to 31 (Top week1 in fig.1.) were pooled together to represent inhibited axillary buds under apical dominance. Positions 30 to 16 in week 1 (Middle week1 fig.1.) contain axillary buds that are further removed from the shoot apex and show outgrowth. Positions 15 to 1 (Bottom week1 fig.1.) represent bottom axillary buds inhibited by correlative inhibition of middle axillary buds. Nodal positions 45 to 42 (Top week2 fig.1.) were pooled because the buds in this section showed the strongest outgrowth after week 2. Nodal positions 41 to 38 (Middle week2 fig.1.) were pooled because these buds showed a diminishing outgrowth after week 2 when compared to the nodal positions above them. Nodal positions 37 to 31 (Bottom week2 fig.1.) were pooled to represent buds that show inhibited outgrowth after week 2. Samples were ground in liquid nitrogen and separate samples were weighed for gene expression analysis (100mg/sample) and UPLC-MS/MS (50mg/sample) .

\section{Gene isolation and Gene expression analysis}

Genes involved in axillary bud outgrowth BRC1 (accession JX870411) and IPT (accession JQ711176) were isolated from Chrysanthemum morifolium. For gene expression analysis, RNA was extracted using a modified CTAB protocol (Luypaert et al, unpublished) and was converted to cDNA using the iScript cDNA Synthesis kit (BioRad). Expression of both branching genes was quantified using RT-qPCR according to De Keyser et al. (2013). Qbase $^{+}$software (Biogazelle) was used for all data analysis. Gene expression was normalised using 3 validated reference genes in stem samples (ACT2, ATUB, UBQ10; reference target stability 0,379) and 3 genes in apex and axillary bud samples ( $A T U B, U B C, E F 1 \alpha$; reference target stability 0,55).

\section{Hormone measurement}

Samples were extracted and analysed by means of UPLC-MS/MS for quantification of IAA and cytokinins according to Prinsen et al. $(1995,2000)$.

\section{RESULTS AND DISCUSSION}

\section{Measurement of axillary bud outgrowth}

The total plant height (length from the base of the plant to the shoot apex ) was (in cm) $39,9 \pm 1,5 ; 53,1 \pm 1,5 ; 60,9 \pm 2,1 ; 61,0 \pm 2,2 ; 61,5 \pm 2,4$ for week1, week2, week3, week4 and week5, respectively. The measurements of axillary bud length are shown in Figure 1. During week1 and week2 plants grew vegetatively. During vegetative growth, the plant displayed apical dominance, represented by the short axillary buds at the higher positions near the apex in week1 (positions 37 to 31 ) and week2 (positions 45 to 38). The middle positions were less influenced by the apex and had longer axillary buds or outgrown shoots (30 to 16 for week1 and 37 to 16 for week2). The lower positions (15 to 1) showed less outgrowth as they were possibly inhibited by correlative inhibition due to the outgrowth of axillary buds above them, a pattern also observed by Chen et al., 2013 and mentioned in other species (Cline 1996; McSteen and Leyser., 2005). After week2, a 
transition to generative growth took place as the shoot apical meristem developed into an inflorescence meristem. At this transition new leaves and axillary buds were no longer initiated at the apex. During the generative growth from week 3 to week 5 a release from apical dominance was observed with a strong outgrowth of the axillary shoots under the apex (positions 47 to 42 ). The lower positions (41 to 38) showed less shoot growth, and the basal positions (37 to 1 ) had even more reduced growth .

The observed axillary shoot growth of this chrysanthemum cultivar corresponds with a control of axillary bud outgrowth by apical dominance in the vegetative growth phase. This is evidenced by the inhibited growth of axillary buds under the apex, the more elongated buds in the middle of the stem and the inhibited outgrowth near the base of the plants. These patterns correspond with previous observations in chrysanthemum cut flower axillary bud growth (Jiang et al., 2012; Chen et al., 2013). The release of apical dominance by the transition to the generative growth phase is accompanied by growth of the axillary shoots directly under the apex. This pattern corresponds to the outgrowth of the first 10 axillary buds under the apex, aswas observed previously in chrysanthemum cut flower after loss of apical dominance by decapitation and floral transition (Chen et al., 2013).

\section{Measurement of auxin (IAA) and cytokinin}

Auxin content in the shoot apex decreased by $60 \%$ from week 1 to week 2 (Fig.2.A.). The IAA content has decreased significantly from week1 to week 2 in the top and middle part of the stem. The IAA content in the bottom stem has decreased less and is higher than in the top and middle part of the stem at week2. In axillary buds there was no remarkable decrease from week 1 to week 2 and IAA levels in the top axillary buds are higher than in the middle and bottom positions (data not shown).

Cytokinin levels in the top stem part increased greatly from week1 to week2. Similarly, in the top axillary buds there was a cytokinin increase from week1 to week2 (data not shown).

We report here a high auxin content in the shoot apex and in the stem during the vegetative growth phase and a decrease of the auxin content preceding bud outgrowth after floral initiation. This corresponds with the hypothesis of an apical dominance where a high auxin production and transport from the vegetative shoot apex inhibits the outgrowth of axillary buds (Chatfield et al., 2000; Wang et al., 2006, Jiang et al., 2012).

In the stem and in the axillary buds of nodal positions directly under the apex, an increased cytokinin content was observed at the transition to generative growth. This corresponded to a strong outgrowth after the release from apical dominance and is consistent with reports of an increased cytokinin content in upper axillary buds at the release from apical dominance (Geuns et al., 2001; Tanaka et al., 2006).

\section{Expression analysis of branching genes}

The relative expression values of $B R C 1$ and IPT3 are given in Fig.3. for axillary buds and the shoot apex samples. In the axillary buds, $B R C 1$ expression is increased from week 1 to week 2 in the bottom position, while the top and middle position show slightly decreased trend(Fig.3.A.). In the stem, $B R C 1$ expression was lower in week 2 compared to week1 in the top, middle and bottom positions (data not shown). The $B R C 1$ expression profile in the axillary buds could indicate that $B R C 1$ is down-regulated in the top axillary buds when they were released from apical dominance; this resulted in bud outgrowth after week2. The bottom axillary buds showed inhibited bud outgrowth after week2, correlating with a higher $B R C 1$ expression. This corresponds with the previous reports of 
high expression in inhibited axillary buds and down-regulation in activated buds (Chen et al., 2013). The down-regulation of $B R C l$ coincides here with the IAA decrease from week1 to week2. This matches with $B R C 1$ acting downstream from auxin in the inhibition of axillary bud outgrowth (Aguilar-Martinez et al., 2007). IPT3 showed low expression in shoot apex and top axillary buds compared to middle and bottom axillary buds (Fig. 3B). From week1 to week2 IPT3 expression decreased significantly. Similar IPT3 expression profiles were observed in stem samples (data not shown). With a decreased IPT3 expression in axillary bud and stem samples from week1 to week2, one would expect a decreased cytokinin biosynthesis. However, increased cytokinin levels were measured in the top axillary buds and stem from week1 to week2. A possible explanation might be that other cytokinin biosynthetic genes beside IPT3 are more important in the cytokinin biosynthesis at the nodal positions. IPT1 and IPT2 for example are associated with increased cytokinin biosynthesis and cytokinin levels after decapitation of pea plants preceding bud outgrowth (Tanaka et al., 2006).

\section{CONCLUSIONS}

The transition from vegetative to generative growth in the studied chrysanthemum cut flower cultivar resulted in a release from apical dominance and the outgrowth of axillary buds under the apex. This outgrowth is preceded by a decreased auxin content in the shoot apex and stem, an increase in cytokinin content in the upper axillary buds and a decreased expression of $B R C l$. Additional to the results presented here we have done further work using measurements and samples of a different chrysanthemum cultivar. Furthermore we have expanded the expression analysis to include the $M A X$ strigolactone biosynthesis and signalling genes and the $L s l$ gene.

\section{ACKNOWLEDGMENTS}

This research was funded by the Institute for the Promotion of Innovation through Science and Technology in Flanders (IWT-Vlaanderen Grant No. 110771). The authors wish to thank Magali Losschaert and Laurence Desmet for outstanding technical assistance during the experiments.

\section{Literature cited}

Aguilar-Martínez, J.A., Poza-Carrión, C. and Cubas, P. 2007. Arabidopsis BRANCHED1 acts as an integrator of branching signals within axillary buds. Plant Cell. 19(2):458-72.

Chatfield, S.P., Stirnberg, P., Forde, B.G. and Leyser, O. 2000. The hormonal regulation of axillary bud growth in Arabidopsis. Cell Mol. Biol. 24(2):159-69.

Chen, X., Zhou, X., Xi, L., Li, J., Zhao, R., Ma, N. and Zhao, L. 2013. Roles of DgBRC1 in regulation of lateral branching in chrysanthemum (Dendranthema xgrandiflora cv. Jinba). PloS One. 8(4), e61717

Cline, M.G. 1991. Apical dominance. Botan. Rev. 57(4):318-358.

Cline, M.G. 1996. Exogenous Auxin Effects on Lateral Bud Outgrowth in Decapitated Shoots. Ann. Botan.. 78:255-266.

De Keyser, E., Desmet, L., Van Bockstaele, E. and De Riek, J. 2013. How to perform RT-qPCR accurately in plant species? A case study on flower colour gene expression in an azalea (Rhododendron simsii hybrids) mapping population. BMC Mol. Biol. 14(1):13. 
Domagalska, M.A. and Leyser, O. 2011. Signal integration in the control of shoot branching. Mol. Cell Biol. 12(4):211-21.

Dong, L., Ishak, A., Yu, J., Zhao, R. and Zhao, L. 2013. Identification and functional analysis of three MAX2 orthologs in chrysanthemum. J. Integ. Plant Biol. 55(5):434-42.

Dun, E.A., de Saint Germain, A., Rameau, C. and Beveridge, C.A. 2012. Antagonistic action of strigolactone and cytokinin in bud outgrowth control. Plant Physiol. 158(1):487-98.

Geuns, J.M.C., Struyf, T., Prinsen, E., Valcke, R. and Onckelen, H. Van. 2001. Apical dominance in Pssu-ipt -transformed tobacco, 58:911-921.

Hayward, A., Stirnberg, P., Beveridge, C. and Leyser, O. 2009. Interactions between auxin and strigolactone in shoot branching control. Plant Phys. 151(1):400-12.

Jiang, B., Miao, H., Chen, S., Zhang, S., Chen, F. and Fang, W. 2009. The Lateral Suppressor-Like Gene, DgLsL, Alternated the Axillary Branching in Transgenic Chrysanthemum (Chrysanthemum $\times$ morifolium) by Modulating IAA and GA Content. Plant Mol. Biol. Rep. 28(1):144-151.

Jiang, B., Chen, S., Jiang, J., Zhang, S., Chen, F. and Fang, W. 2012. Changes of endogenous hormones in lateral buds of chrysanthemum during their outgrowth. Russian Journal of Plant Physiol. 59(3):356-363.

Khodakovskaya, M., Vanková, R., Malbeck, J., Li, A., Li, Y. and McAvoy, R. 2009. Enhancement of flowering and branching phenotype in chrysanthemum by expression of ipt under the control of a $0.821 \mathrm{~kb}$ fragment of the LEACO1 gene promoter. Plant Cell Rep. 28(9):1351-62.

Leyser, O. 2009. The control of shoot branching: an example of plant information processing. Plant Cell Envir. 32(6):694-703.

Liang, J., Zhao, L., Challis, R. and Leyser, O. 2010. Strigolactone regulation of shoot branching in chrysanthemum (Dendranthema grandiflorum). J. Exp. Bot. 61(11):3069-78.

McSteen, P. and Leyser, O. 2005. Shoot branching. Annual Review of Plant Biology, 56:353-74.

Müller, D. and Leyser, O. (2011). Auxin, cytokinin and the control of shoot branching. Ann. Bot. 107(7):1203-12.

Prinsen, E., Redig, P., Van Dongen, W., Esmans, E. L. and Van Onckelen, H. 1995. Quantitative analysis of cytokinins by electrospray tandem mass spectrometry. Rapid Comm. Mass Spectrom. 9:948-953.

Prinsen, E., Van Laer, S., Öden, S. and Van Onckelen, H. 2000. Auxin Analysis. Plant Hormone Protocols, 49-65.

Shinohara, N., Taylor, C. and Leyser, O. 2013. Strigolactone can promote or inhibit shoot branching by triggering rapid depletion of the auxin efflux protein PIN1 from the plasma membrane. PLoS Biol. 11(1).

Tanaka, M., Takei, K., Kojima, M., Sakakibara, H. and Mori, H. 2006. Auxin controls local cytokinin biosynthesis in the nodal stem in apical dominance. Cell Mol. Biol. 45(6):1028-36.

Thimann K.V., Skoog F. and Kerckhoff W.G. 1934. On the inhibition of bud development and other functions of growth substance in Vicia faba. Proc. R. Soc. Lond. B. Biol. Sci. 114(798):317-339.

Waldie, T., McCulloch, H. and Leyser, O. 2014. Strigolactones and the control of plant development: lessons from shoot branching. Cell Mol. Biol. 607-622. 
Wang, G., Römheld, V., Li, C. and Bangerth, F. 2006. Involvement of auxin and CKs in boron deficiency induced changes in apical dominance of pea plants (Pisum sativum L.). J. Plant Physiol. 163(6):591-600.

\section{Figures}

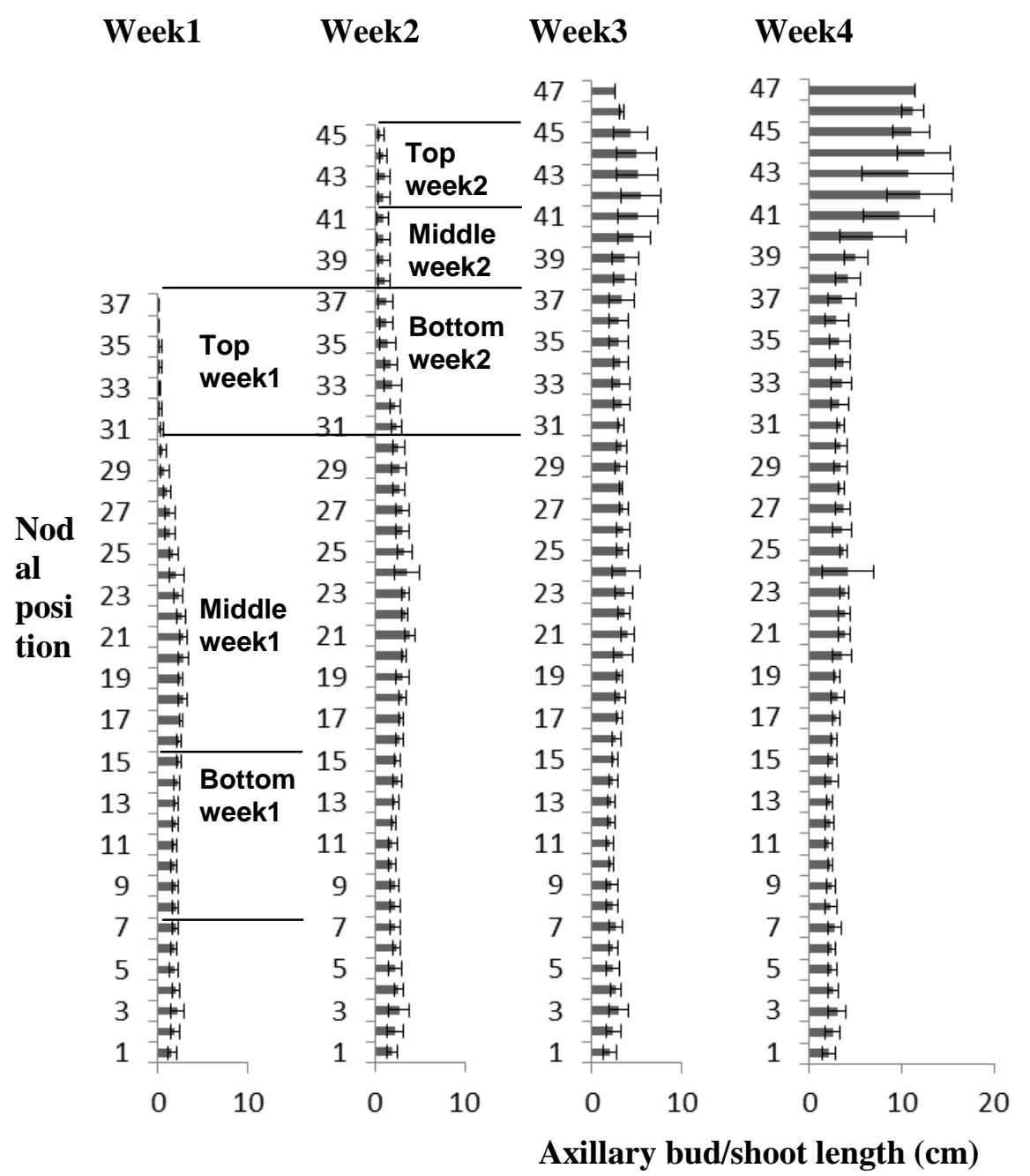

Week5

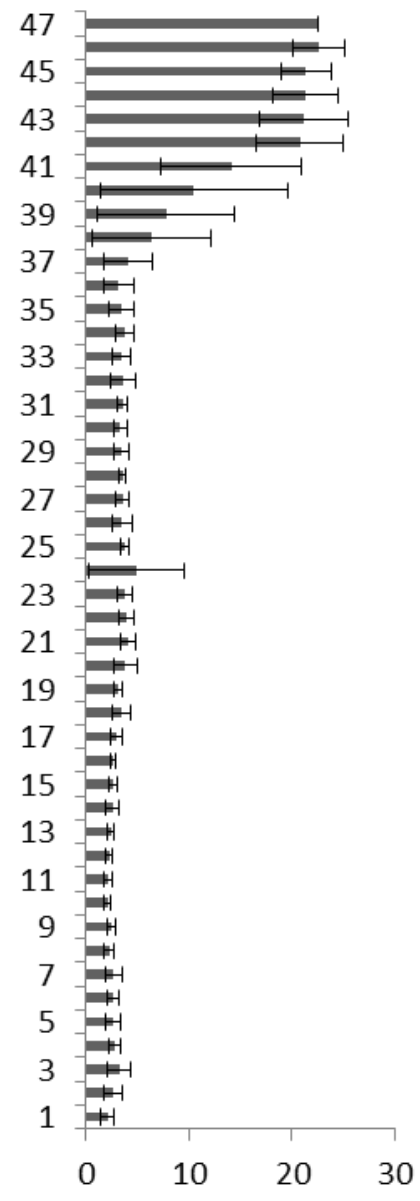

Figure 1. Axillary bud/shoot length of Chrysanthemum cultivar C17. Mean bud/shoot length $( \pm S E ; n=10)$ is shown for the nodal positions presented on the $y$-axis with 1 being the node closest to the base of the plant. For week1 and week2, the nodal positions for which samples of stem and axillary buds were pooled together for gene expression analysis and hormone measurement (Top week1, Middle week1, Bottom week1, Top week2, Middle week2, Bottom week2) are indicated. 

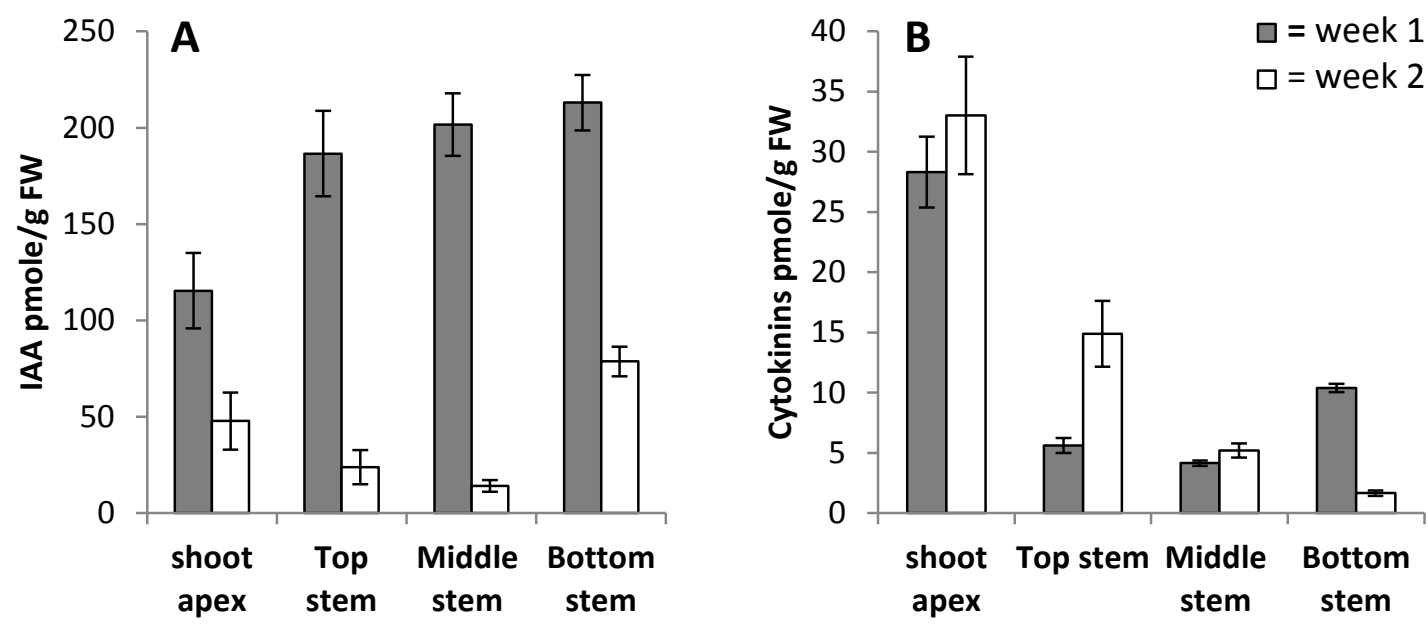

Figure 2. Hormone measurement in week1 and week2. (A) the IAA content of the shoot apex and stem samples. (B) the total measured cytokinin content of the shoot apex and stem samples. Mean values $(n=3) \pm S E$ are indicated.
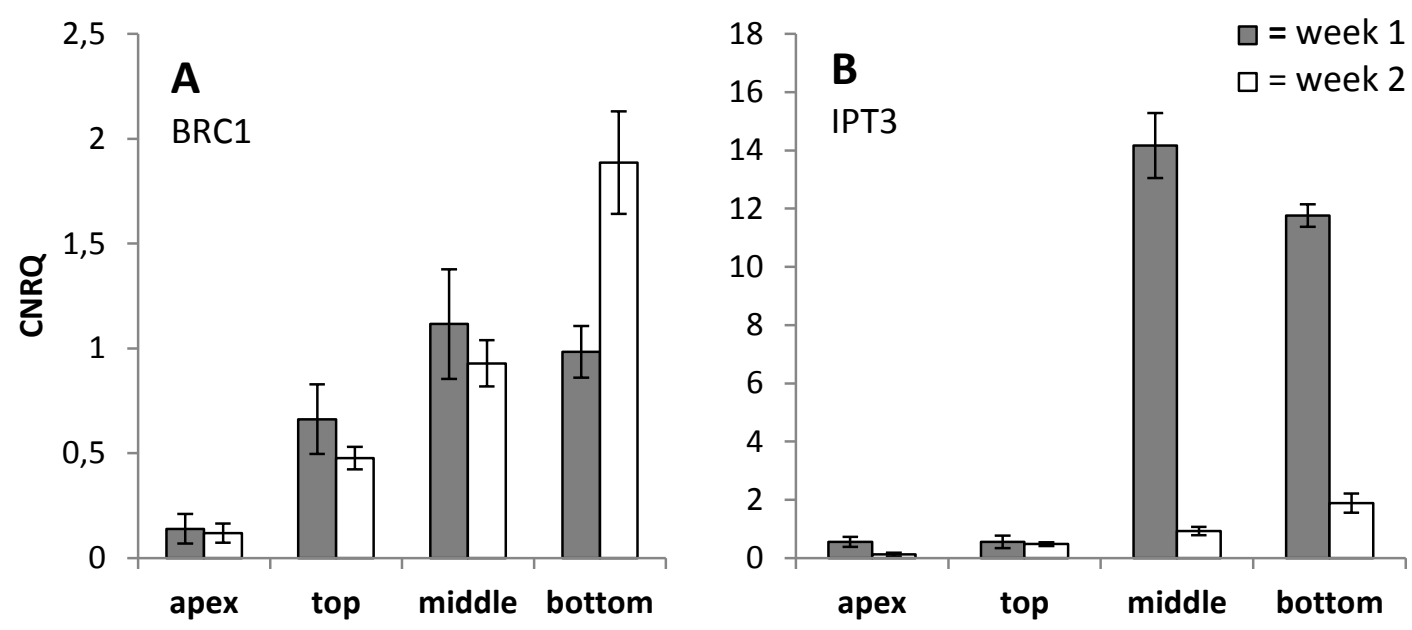

Figure 3. RT-qPCR expression analysis in week1 and week2. BRC1 (A) and IPT3 (B) expression in axillary buds and shoot apex. Expression levels are given as CNRQvalues (Calibrated Normalized Relative Quantities). Mean values $(n=3) \pm S E$ are indicated. 